\title{
Unsteady CFD Analysis of a Delta Wing Fighter Configuration by Delayed Detached Eddy Simulation
}

\author{
Heinrich Lüdeke ${ }^{1}$, Stefan Leicher ${ }^{2}$ \\ ${ }^{1}$ DLR, Institute of Aerodynamics and Flow Technology \\ Lilienthalplatz 7, D-38108 Braunschweig \\ e-mail: heinrich.luedeke@dlr.de \\ ${ }^{2}$ EADS-MAS Germany GmbH, \\ Willy-Messerschmitt-Strasse, D-85521 Ottobrunn
}

\begin{abstract}
While the flow physics of generic delta wings with sharp leading edges are largely understood, realistic configurations with rounded leading edges and canards are still of scientific and industrial interest. The goal of the presented study is the investigation of such a realistic delta wing configuration at $15^{\circ}$ angle of attack and at high Reynolds number in comparison with detailed wind tunnel measurements. Former studies have shown the superior results of large and Detached-eddy simulations (DES) for delta wings in comparison with RANS computations. The original standard formulation of DES has shown the drawback of only grid based prediction of the boundary layer edge. To overcome this deficiency the technique of Delayed DES (DDES) was developed some years ago. This new model is based on a simple modification of the original formulation to provide a dependency of the RANS-LES switch on turbulent flow properties. The numerical DES and DDES results are compared with data from the TU Munich wind tunnel facility. Comparison of statistical data as well as velocity spectra in the flow field with experiments will be presented.
\end{abstract}

\section{Introduction}

While the flow physics of generic delta wings with sharp leading edges is largely understood and well described in the literature (Morton 2002) realistic configurations with rounded leading edges and canards are still of scientific and industrial interest.

The goal of the presented study is the investigation of such a realistic fighter configuration by recent turbulence models, a comparison with former numerical studies and validation by detailed wind tunnel measurements of the TU-Munich (Breitsamter 1997). Former studies have shown the superior results of large and Detached-eddy simulations (DES) for delta wings in comparison with RANS computations (Morton 2002). DES is a hybrid approach for the modelling of turbulent flow fields at complex geometries. The idea is to combine the best features of both, the Reynolds-averaged Navier-Stokes (RANS) and the large eddy simulation (LES) approach. The initial formulation of DES, denoted DES97 in (Spalart 2006) has shown ambiguous behaviour for extended boundary layers if 
tangential grid spacing parallel becomes inappropriate due to only grid dependent RANS-LES switching. To overcome this deficiency the technique of Delayed DES was developed in recent publications (Spalart 2006). The new model is based on a simple modification of the original formulation to provide a dependency of the RANS-LES switch on turbulent flow properties. For all computations the Spalart Allmaras one equation turbulence model is used as an accurate and efficient base for DES. All simulations were carried out by the unstructured DLR Tau code which is validated in various scientific and industrial cases (Schwamborn 2006).

The described test case was investigated by different research groups with structured as well as unstructured codes and grids (Gurr 2006, Rieger 2005). Due to open questions concerning the validation with experimental data, the present study shows an investigation with the new formulation of the delayed DES was carried out on an unstructured grid provided by EADS-MAS. The results are compared with wind tunnel data from the wind tunnel facility of the TU Munich and former investigations of EADS-MAS by the standard DES97 model. Comparisons of statistical data as well as velocity spectra in the flow field with experiments will be presented. A snapshot of the DDES result is shown in Fig. 1 with an iso-vorticity contour surface coloured by the pressure distribution.

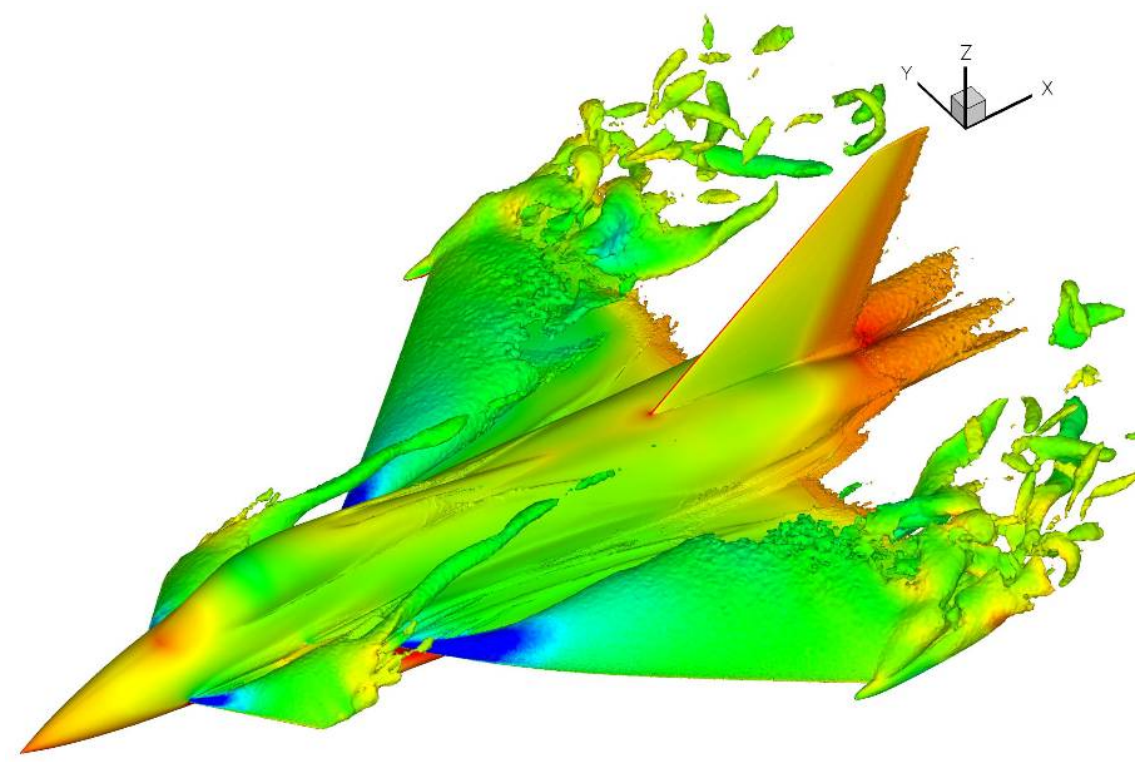

Figure 1 Vorticity Iso-surfaces coloured by $\mathrm{Cp}$ distribution of the generic FA-5 fighter configuration at $15^{\circ} \mathrm{AoA}$ (DDES result). 


\section{Numerical tools}

\subsection{Description of the flow solver}

All CFD computations for this study are performed by the hybrid structured/unstructured DLR-Navier-Stokes Solver Tau (Gerhold 1997). The Taucode is a second order finite-volume flow solver for the Euler and Navier-Stokes equations in the integral form (Schwamborn 2006). Different numerical schemes like cell-centered for sub- and transonic flow and AUSMDV for super- and hypersonic flow conditions are implemented. A three-stage explicit Runge-Kutta scheme and a point implicit LUSGS scheme are options to advance the solutions in time for steady flow fields. For convergence acceleration local time stepping, implicit residual smoothing and full multigrid are implemented. Fast and accurate transient flow simulations are computed by a Jameson type dual time step-ping scheme, as an implicit algorithm which is not restricted in the choice of the smallest timestep in the flow field (Gerhold 1997). Within this inner loop of the dual time stepping scheme all mentioned acceleration techniques are applicable. Several one- and two equation turbulence models are available for steady as well as unsteady RANS simulations.

\subsection{DES97}

In the following a short description of the standard DES formulation as a hybrid RANS-LES technique, developed by Spalart and Shur in 1997 will be given (Spalart 1997). The one-equation Spalart-Allmaras (SA) model, briefly described in the following, is used as a base for the original DES formulation. The model defines the eddy viscosity field as

$$
\mu_{t}=\rho v_{t}=\rho \tilde{v} f_{v 1}
$$

with $\rho$ as the density, $v_{t}, \mu_{t}$ as the turbulent kinematic and dynamic viscosity and $f_{v 1}$ as a near wall-function that guarantees linear behaviour of the turbulent transport quantity in the vicinity of walls. The auxiliary wall functions are described in detail in the original work of Spalart (Spalart 1994). The distribution of the transport quantity $\widetilde{v}$ is determined by the solution of

$$
\frac{D(\rho \tilde{v})}{D t}=\underbrace{c_{b 1} \tilde{S} \rho \tilde{v}}_{P}-\underbrace{C_{w 1} f_{w} \rho\left(\frac{\tilde{v}}{d}\right)^{2}}_{D}+\underbrace{\frac{\rho}{\sigma}\left\{\nabla[(v+\tilde{v}) \nabla \tilde{v}]+c_{b 2}(\nabla \tilde{v})^{2}\right\}}_{D F}
$$

with $\mathrm{d}$ as the wall distance. This transport equation contains phenomenological models of production $P$, destruction $D$ and diffusion $D F$. The destruction term $D$ is necessary for the modelling of blocking effects near walls. In the production term $P$ a modified vorticity $\widetilde{S}$ appears that maintains the linear behavior of the model near walls. Additional wall functions like $f_{w}$ are used to maintain linear behaviour of all quantities near walls. Their construction and the associated settings of the model constants can be found in (Spalart 1994).

The original approach of DES bases on a modification of the wall distance term in the SA model to switch the standard RANS model into an LES mode. To keep the preferred features of both approaches, RANS is used in the unsteady boundary 
layer flow with a standard grid resolution where it performs reasonable results while LES is used in separated regions where relevant turbulent scales can be resolved. The switching between RANS and LES bases on a characteristic length scale, chosen to be proportional with $\Delta$ which is the largest cell dimension:

$$
\Delta=\max (\Delta x, \Delta y, \Delta z)
$$

For the standard DES formulation the wall distance $d$ in the SA model is replaced by $\widetilde{d}$ where $\widetilde{d}$ is defined as:

$$
\widetilde{d}=\min \left(d, C_{D E S} \Delta\right)
$$

with $C_{D E S}$ as a constant calibrated by using the decay of isotropic turbulence. In this mode a local equilibrium between production and destruction term in the SA model is expected. This local balance leads to the relation $\widetilde{v} \propto \widetilde{S} \cdot \widetilde{d}^{2}$ which is very similar to the relation in the Smagorinsky model.

\subsection{Delayed DES}

One equation models such as S-A do not have a natural turbulent length scale based upon the turbulent flow field like two equation models but involve the parameter $r$ which is also the squared ratio of a model length scale to the wall distance $\mathrm{d}$. This length scale is not internal in that it involves the mean shear rate. For DDES the parameter $r$, used by the SA model as an indicator in the near wall function, is slightly modified in order to apply to any eddy viscosity model and be slightly more robust in irrotational regions

$$
r_{d} \equiv \frac{\widetilde{v}}{\sqrt{U_{i, j} U_{i, j}} \kappa^{2} d^{2}}
$$

where $U_{i, j}$ are the velocity gradients and $d$ the distance to the wall Similar to $r$ in the SA model. This parameter equals 1 in a logarithmic layer and falls to 0 gradually towards the edge of the BL. The use of $\widetilde{v}$ remains linear near walls and ensures furthermore that $r_{d}$ remains everywhere away from 0 . The subscript $d$ represents delayed. The quantity rd is used in functions such as:

$$
f_{d} \equiv 1-\tanh \left(\left[8 r_{d}\right]^{3}\right)
$$

which are designed to be 1 in the LES region where $r_{d}<<1$ and 0 elsewhere. It is furthermore designed to be rather steep near $r_{d}=0.1$. The values 8 and 3 for the constants are taken from (Spalart 2006). These values for the coefficients ensure that the solution is essentially identical to the RANS solution in BL regions.

The application of the described procedure to the S-A based DES proceeds by redefining the DES length scale $d$ :

$$
\widetilde{d} \equiv d-f_{d} \max \left(0, d-C_{D E S} \Delta\right)
$$

Setting $f_{d}$ to 0 yields RANS $\widetilde{d}=d$ while setting it to 1 gives DES97 (see last section) 
3. Flow conditions and computational grid

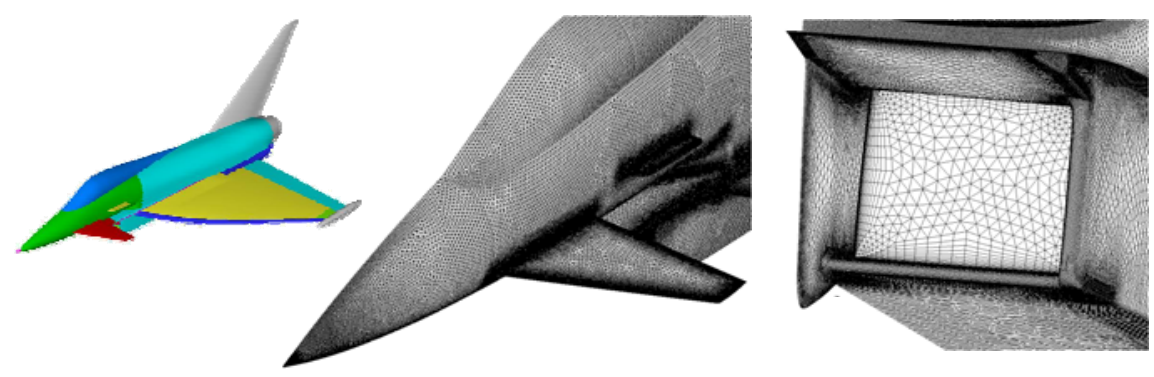

Figure 2 Details of the surface grid for the FA-5 fighter configuration.

The computations are carried out under subsonic conditions at a moderate angle of attack in order to recreate the experimental conditions of the TU Munich wind tunnel facility where detailed experimental data was generated (Breitsamter 1997). The flow conditions are Mach number 0.125 and Reynolds-number $3 \cdot 10^{6}$ at $15^{\circ}$ angle of attack at these conditions preconditioning was added to reduce numerical problems with incompressible flow.

An unstructured grid of 11.6 million nodes with 27 wall normal prism layers for boundary layer resolution and grid refinements in critical regions was used for all simulations. Aside of the in- and outflow conditions in the far field, exhaust and nozzle conditions of the same type are prescribed at in- and outlet of the fighter. Parts of the surface grid are shown in Fig. 2 while the influence of the field sources upon the grid densities for $20 \%$ and $90 \%$ wing chord are visible in Fig. 3.
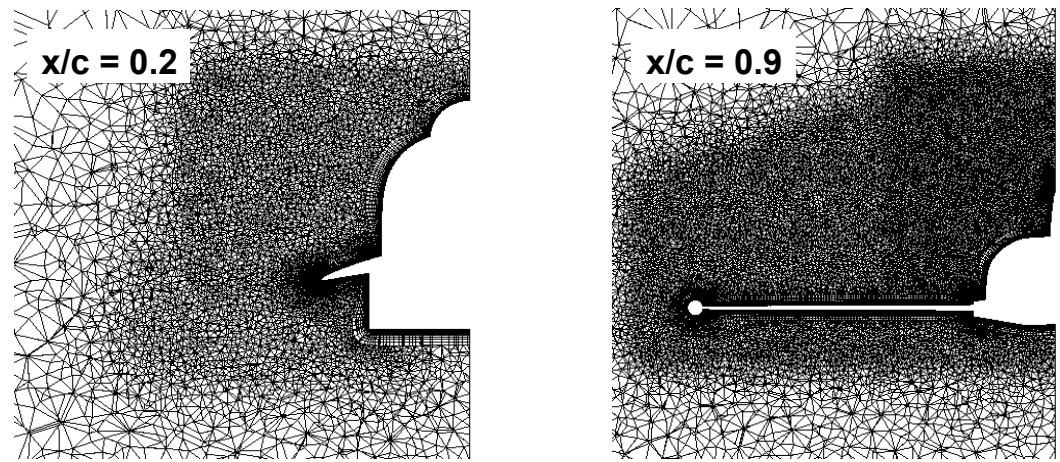

Figure 3 Field sources of the tetrahedral grid around wing and fuselage.

\section{DES97 and Delayed DES results}

For the unsteady computations timesteps in a range between $1 \cdot 10^{-4}$ and $5 \cdot 10^{-4}$ where chosen. This approximate range of the timesteps is computed by the standard technique of dividing the average grid length scale in the region of interest by local velocities. 


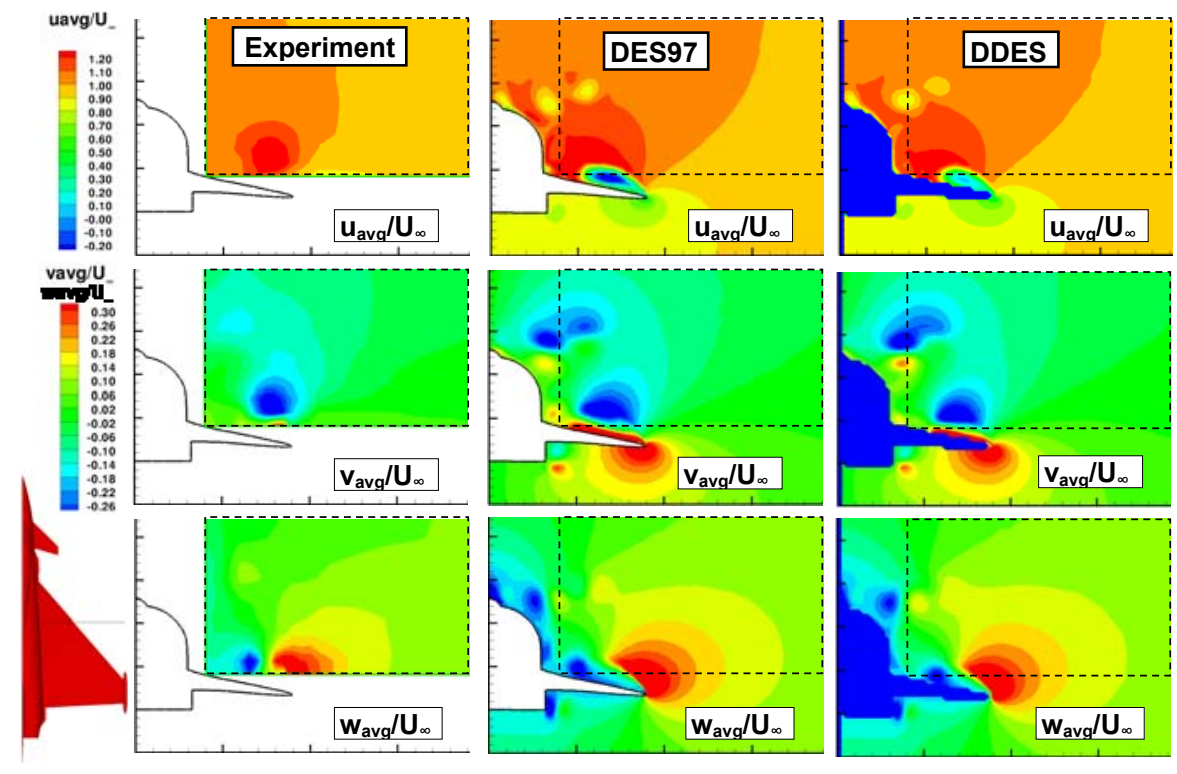

Figure 4 Normalized averaged streamwise velocity components at $40 \%$ wing chord. Left: Experiment, middle: DES97, right: DDES.

Top: $\mathrm{u}_{\text {avg }} / \mathrm{U}_{\infty}$, middle: $\mathrm{v}_{\text {avg }} / \mathrm{U}_{\infty}$, Bottom: $\mathrm{w}_{\text {avg }} / \mathrm{U}_{\infty}$.

For the DES97 simulations, which were carried out first, the larger timestep of $5 \cdot 10^{-4} \mathrm{~s}$ was chosen, while for DDES a finer temporal resolution of $1 \cdot 10^{-4}$ seemed to be more appropriate. Furthermore the recommended Low-Reynolds-Number correction of Strelets was included for the DDES simulations (Spalart 2006).

The DDES results were interpolated for post-processing on a rectangular mesh with a similar resolution used for the measurements. These interpolated datasets are shown in all contourplots, even if the fighter contour looks coarse grained.

The velocity components at 40\% wing chord, averaged over approximately $1 \mathrm{sec}$, are shown in Fig. 4. The dashed box in the computational results represents the area of experimental data, shown in the first column. As visible the DES97 and DDES results agree widely with the experimental dataset. Differences appear especially for the v-component, for which an over-prediction of the canard-vortex is obvious. The influence is weaker for the $\mathrm{u}$ - and $\mathrm{w}$-components, but still evident. Another difference between CFD and experiment becomes obvious for the $\mathrm{u}-$ component in Fig. 5. No negative values were investigated in the wind tunnel investigations for this quantity, while both DES versions predict negative velocities in the core region of the vortex. The same behaviour was investigated in former studies by the TU-Berlin (Gurr 2006). It is likely, that an insufficient resolution of the very small turbulent structures in this region is responsible for this effect. Nevertheless, for the DDES simulations the negative velocities are less pronounced. V- and w components show for both DES-types good agreement with the wind-tunnel data. 

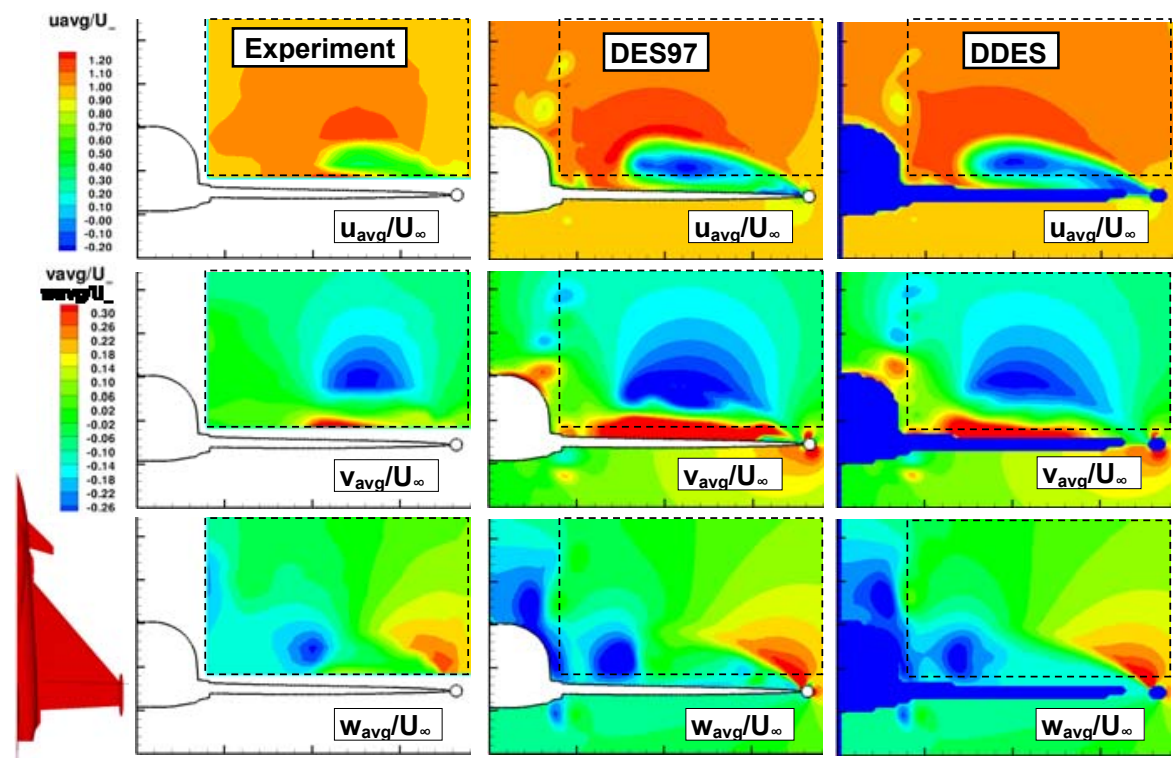

Figure 5 Normalized averaged streamwise velocity components at $80 \%$ wing chord. Left: Experiment, middle: DES97, right: DDES.

Top: $\mathrm{u}_{\text {avg }} / \mathrm{U}_{\infty}$, middle: $\mathrm{v}_{\text {avg }} / \mathrm{U}_{\infty}$, Bottom: $\mathrm{w}_{\text {avg }} / \mathrm{U}_{\infty}$.

In Fig. 6 and 7 the standard deviations of the velocity-components are compared with the wind tunnel data. The agreement of both DES types with the measurements is at least reasonable for the cut at $40 \%$ wing chord (Fig. 6). Especially for DDES the unsteadiness inside the boundary layer is less pronounced, which is an appropriate behaviour for the RANS region under the physical assumptions made for the RANS model. Nevertheless it prevents the LES region from developing the instabilities near the wing tip, appropriate for a physical behaviour. Especially for the standard-deviation of the w-component, which is as the wall-normal component most influenced by the differences in the DDES method, by far lower values than in the DES97 formulation are visible.

Differences between DES97 and DDES are much more pronounced for the fully separated flow at the $80 \%$ cut (Fig. 7). In this cut DES97 generally overpredicts the standard deviations of all components. Here the DDES results represent the experimental data, again with the exception of the leading edge vortex, which is still overpredicted by the simulations. 


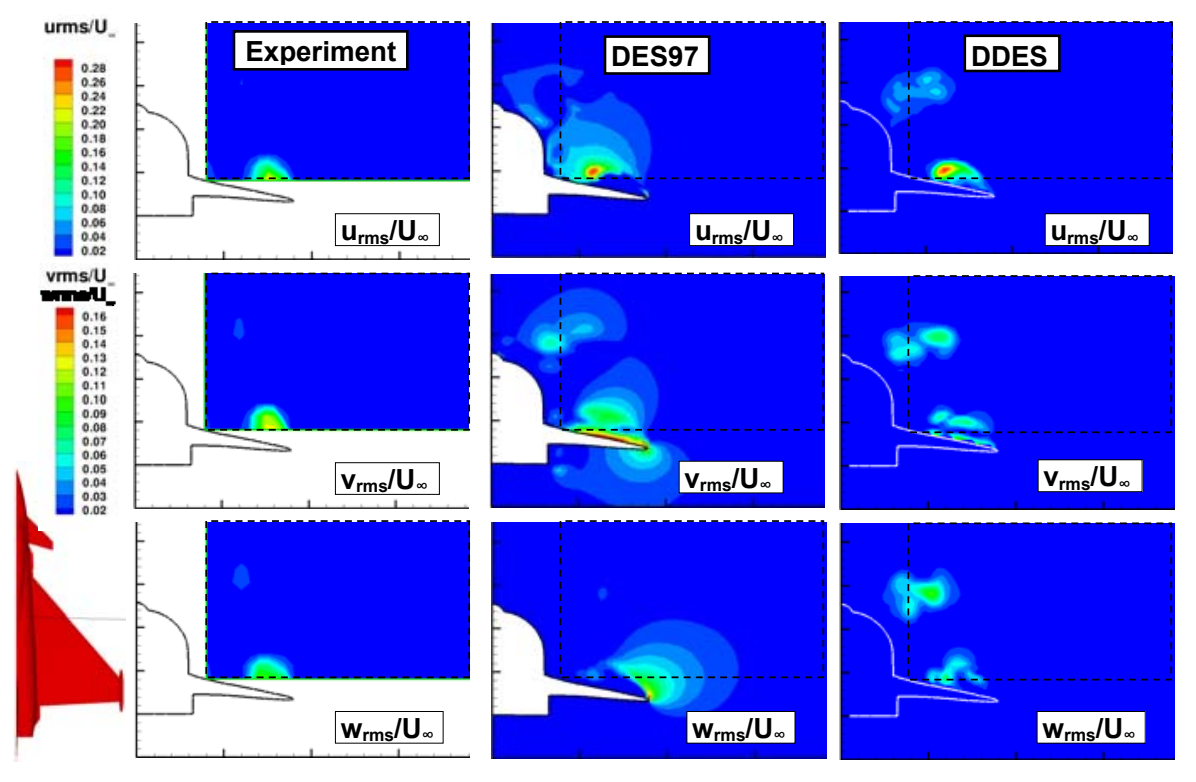

Figure 6 Normalized standard-deviation of streamwise velocity components at $80 \%$ wing chord. Left: Experiment, middle: DES97, right: DDES. Top: $\mathrm{u}_{\mathrm{rms}} / \mathrm{U}_{\infty}$, middle: $\mathrm{v}_{\mathrm{rms}} / \mathrm{U}_{\infty}$, Bottom: $\mathrm{w}_{\mathrm{rms}} / \mathrm{U}_{\infty}$.
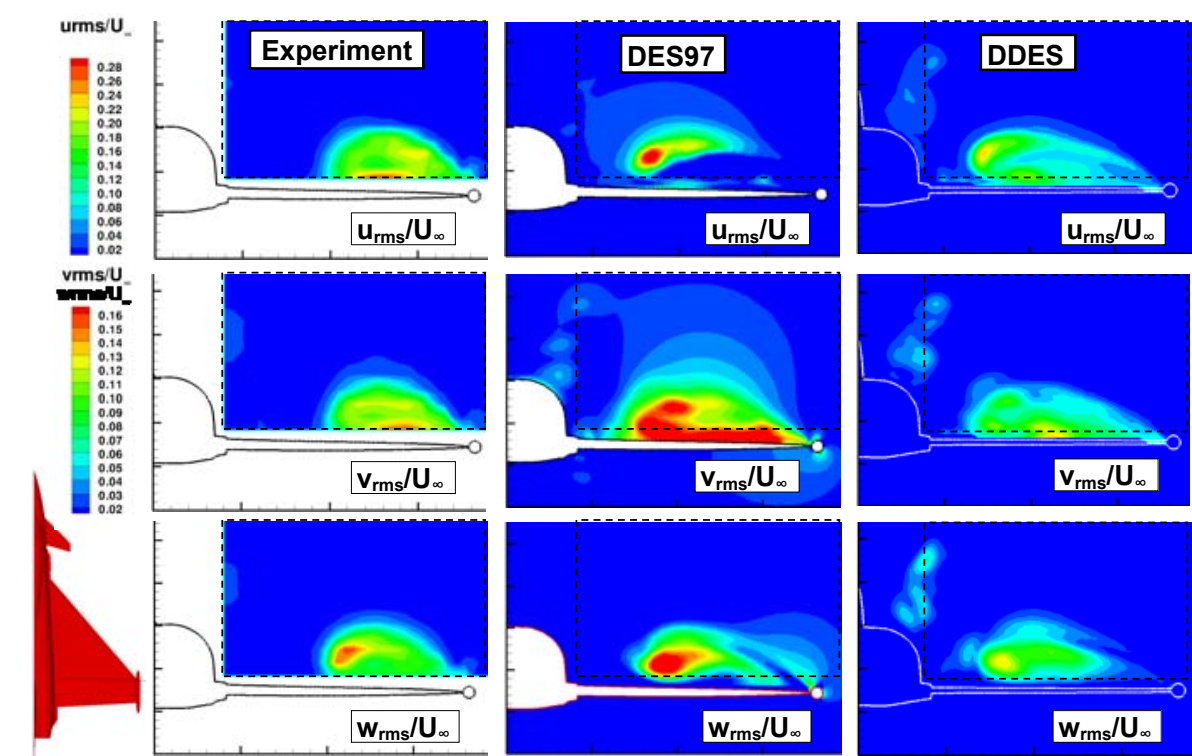

Figure 7 Normalized standard-deviation of streamwise velocity components at $80 \%$ wing chord. Left: Experiment, middle: DES97, right: DDES.

Top: $\mathrm{u}_{\mathrm{rms}} / \mathrm{U}_{\infty}$, middle: $\mathrm{v}_{\mathrm{rms}} / \mathrm{U}_{\infty}$, Bottom: $\mathrm{w}_{\mathrm{rms}} / \mathrm{U}_{\infty}$. 
Fig. 8 shows the power spectral densities of the velocity components for two different points at $90 \%$ wing chord. Especially for point 19, which is located in the middle of the wing, the characteristic peaks are located at the right frequencies. The overprediction of the spectral densities is typical for DES simulations. It is furthermore obvious, that the drop of the spectra for larger frequencies is too steep. This is partly caused by the larger timestep size and may be also attributed to numerical and model dissipation, which have to be investigated in further studies. It is less pronounced for the DDES simulations, which is likely a result of the smaller timestep chosen for this computation.
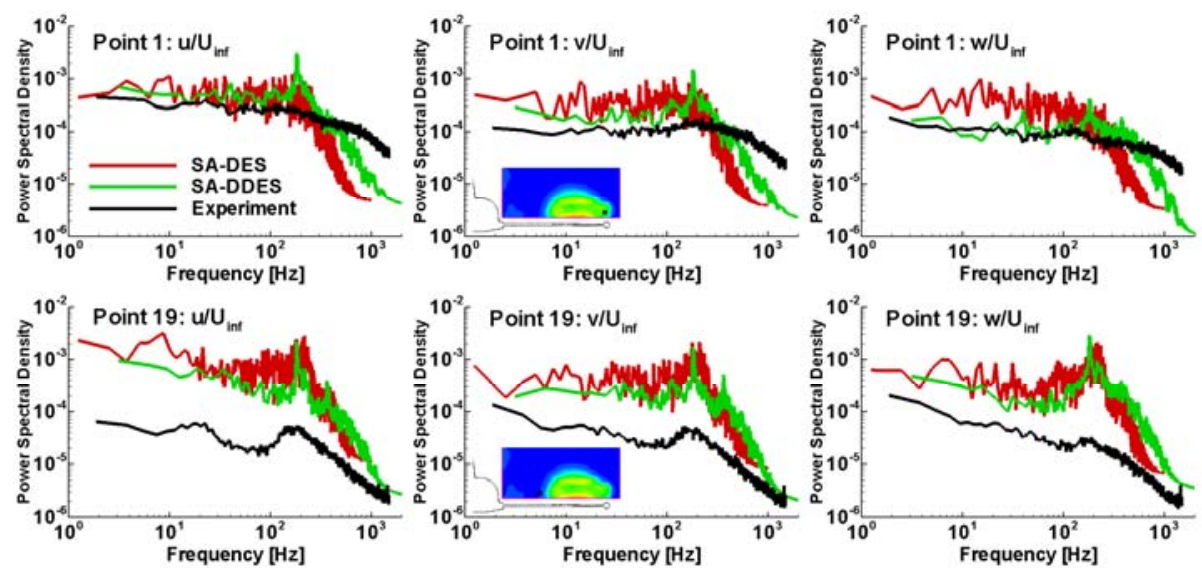

Figure 8 Normalized power spectral densities of velocity components at $90 \%$ wing chord. Cuts in the middle column: $\mathrm{u}_{\mathrm{rms}}$ and position where frequency data is extracted.

Finally the aerodynamic coefficients are plotted over the physical time for DES and DDES in Fig. 9. Unfortunately no experimental data is available for these quantities, so only numerical results are compared. After some start-up time the averages of lift are nearly identical while for drag and pitching moment visible differences appear. The relative differences are about $0.03 \%$ for $C_{L}, 0.6 \%$ for $C_{D}$ and $6.8 \%$ for $\mathrm{C}_{\mathrm{MY}}$. This is still in the range of expected tolerances by taking into account the differences in both simulations.
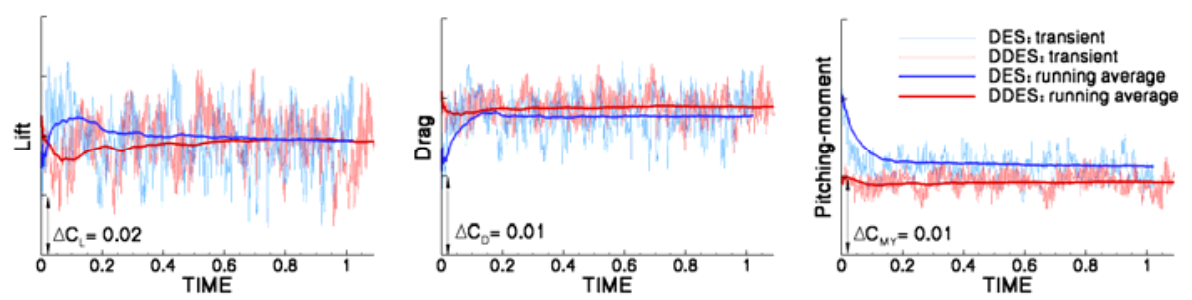

Figure $9 C_{L}, C_{D}$ and $C_{M Y}$ coefficients and running average over time. 


\section{Conclusions}

Detached Eddy Simulations for different variations of a generic FA-5 fighter configuration, as a realistic test case of industrial relevance, are carried out. Comparisons with measurements at the wind tunnel facility TU Munich have shown good agreement, though both modelling approaches were not able to resolve all feature of the experimental data base.

A globally better resolution of unsteady effects by the recent formulation of Delayed DES was found for the investigated configuration. Improvements for this kind of configurations are necessary in areas of rounded leading edges, where an under-prediction of unsteady flow behaviour is investigated at the beginning of the LES zone. Globally the application of both DES versions on the given grid could be justified by the present investigation.

\section{Acknowledgments}

The authors have to thank Yury Ergorov from Ansys Inc for valuable discussions and C. Breitsamter for providing kindly the experimental database. Furthermore the TU Berlin for the fourier transformation tool dftavg.

\section{References}

Morton, S.A, Forsythe, J.R., Mitchell, A.M., Hajek, D. (2002): DES and RANS Simulations of Delta Wing Vortical Flows. AIAA 02-0587, 2002.

Breitsamter, C. (1997): Turbulente Strömungsstrukturen an Flugzeugkonfigurationen mit Vorderkantenwirbeln. Dissertation, Tecnical University Munich, 1997.

Spalart, P.R., Deck, S., Shur, M.L. et.al. (2006): A New Version of Detached-Eddy Simulation, Resistant to Ambiguous Grid Densities. Theoretical and Computational Fluid Dynamics, Volume 20, Number 3.

Schwamborn, D., Gerhold, T., Heinrich, R. (2006): The DLR TAU-code: Recent Applications in Research and Industry. European Conference on Computational Fluid Dynamics, Egmaond aan Zee, ECCOMAS CFD 2006.

Gurr, A., Rieger, H., Breitsamter, C., Thiele, F. (2006): Detached-eddy simulation of the delta wing of a generic aircraft configuration. New Results in Numerical and Experimental Fluid Mechanics V, Notes on Numerical Fluid Mechanics and Multidisciplinary Design, Vol. 92, pp. 463-470, Springer Verlag, 2006.

Rieger, H. (2005): First Experiences with Detached-Eddy Simulation in the Aeronautics Industry. Symposium on Hybrid RANS-LES Methods, Stockholm, Sweden, 14-15 July 2005.

Gerhold, T., Friedrich, O., Evans, J., Galle, M. (1997): Calculation of Complex ThreeDimensional Configurations Employing the DLR-tau-Code. 35th Aerospace Sciences Meeting, Reno, Nevada, January 6-10, 1997.

Spalart, P.R., Jou, W.H., Strelets, M., Allmaras, S.R.(1997): Comments on the feasibility of LES for wings and on a hybrid RANS_LES approach First AFOSR International Conference on DNS/LES, Aug. 4-8, 1997, Ruston, Louisiana.

Spalart, P.R., Allmaras, S.R. (1994): A One Equation Turbulence Transport Model for Aerodynamic Flows, La Recherche Aérospaciale, No. 1, 1994, pp. 5-21. 